\title{
Instantons and Monopoles in the Maximally Abelian Gauge.
}

\author{
A. Harttand M. Teper. \\ Theoretical Physics, University of Oxford, 1 Keble Road, Oxford, OX1 3NP, U.K.
}

November 10, 1995.

\begin{abstract}
We study the Abelian projection of $S U(2)$ instantons in the Maximally Abelian gauge. We find that in this gauge an isolated instanton produces a closed monopole loop within its core and the size of this loop increases with the core size. We show that this result is robust against the introduction of small quantum fluctuations. We investigate the effects of neighbouring (anti)instantons upon each other and show how overlapping (anti)instantons can generate larger monopole loops. We find, however, that in fields that are typical of the fully quantised vacuum only some of the large monopole loops that are important for confinement have a topological origin. We comment on what this may imply for the role of instantons in confinement and chiral symmetry breaking.
\end{abstract}

Oxford Preprint Number: OUTP-95-44-P

hep-lat/9511016

*e-mail: harta@thphys.ox.ac.uk 


\section{Introduction}

The idea of 't Hooft [1] that confinement in non-Abelian gauge theories might be associated with monopoles in suitable Abelian projections of the fields, has been the subject of extensive numerical investigation in recent years. This has been largely due to the observation [2] that in the Maximally Abelian gauge [3] the string tension one obtains from the Abelian Wilson loops appears to equal the full non-Abelian string tension [4].

Recently it has been shown analytically [5] that if one puts a particular classical instanton potential into this gauge then one finds a monopole whose world line passes through the centre of the instanton. This is interesting since large monopole loops contribute to (Abelian) confinement; but it is puzzling because it is known that a reasonably decorrelated ensemble of (anti)instantons does not contribute to non-Abelian confinement. At the same time, because instantons probably contribute to chiral symmetry breaking [6] [7], this raises the possibility of learning something about the connection between chiral symmetry breaking and confinement.

The results of [5] were obtained starting with an $S U(2)$ instanton in a particular gauge and in an infinite volume. However it is well known that the details of the monopole 'gas' differ for the different Gribov copies of a given field. So the first question we would like to answer is whether the analytic result of [5] is typical of all these Gribov copies, or whether it is effectively of measure zero. Since the vacuum contains a 'gas' of topological charges we would also like to know how the presence of neighbouring charges affects the monopole properties of an instanton. Finally, we obviously need to know how robust are our results against the inclusion of quantum fluctuations about the instantons.

To answer these questions we shall discretise instantons on hypercubic periodic lattices. We shall go to the Maximally Abelian gauge numerically and will then study the monopole content of the resulting Abelian fields. We add quantum fluctuations to a lattice instanton by performing a sequence of Monte Carlo steps with the instanton as the initial configuration.

The purpose of our calculations is to tell us how instantons contribute to the gas of monopoles, when one performs the Maximally Abelian projection of $S U(2)$ fields in which the instantons are the only non-perturbative fluctuations. This is a well-defined and tractable problem and its solution seems to us to be a prerequisite to answering the much more ambitious (although probably ambiguous) question of how instantons contribute to the monopole gas in the fully quantised vacuum. To provide the reader with an indication of what this latter problem entails we finish the paper by taking a few typical Monte Carlo generated field configurations and calculate the corresponding monopole distributions. We then cool these fields, gradually exposing the topological charge [8] therein. We calculate the monopole distributions corresponding to these smoothened fields and compare them to those of the original fields and see if there are any correlations with the topological charges in those fields. 


\section{Single Instantons}

To construct an instanton [9] on a discretised hypertorus we follow the procedure in [10]. We begin with the gauge potential

$$
A_{\mu}=\frac{x^{2}}{x^{2}+\rho^{2}} g^{-1}(x) \partial_{\mu} g(x)
$$

where

$$
g(x)=\frac{x_{0} \mathbf{1}+i x_{j} \sigma_{j}}{\sqrt{x^{2}}}
$$

This corresponds to an instanton of size $\rho$ in a particular gauge and in an infinite volume. To construct a corresponding lattice field we define the link variables by

$$
U_{\mu}(n)=\mathcal{P} \exp \left(i \int_{n}^{n+\widehat{\mu}} d x_{\nu} A_{\nu}(x)\right)
$$

(Note that this implies that $\rho$ is in units of the lattice spacing.) In practice we calculate this by the product of $N$ discrete elements

$$
U_{\mu}(n)=\prod_{m=0}^{N-1} \exp \left(\frac{i}{N} A_{\mu}\left(n+\frac{m}{N} \widehat{\mu}\right)\right)
$$

where $\mathrm{N}$ is chosen sufficiently large for this to be an accurate approximation. (For the particular solution we are using eqn(3) can in fact be evaluated analytically.) We now have an approximate lattice instanton but it is in an infinite volume. Since the fields go to different values as $x^{2} \rightarrow \infty$ in different directions, we cannot simply take some reasonably large volume centered on the instanton and impose periodic boundary conditions on it. (If we do so then we find that the mismatch between the fields at the boundary typically carries a topological charge that cancels that of our instanton - so that the total field configuration in effect possesses zero charge.) We therefore apply to the lattice field the gauge transformation $g^{\dagger}(n)$, and this produces a potential that goes to $A_{\mu}(x)=0$ and hence $U_{\mu}(n)=1$ at $x^{2}=\infty$. (In doing so we have gone to a singular gauge. For numerical reasons it is better to do so after discretising the instanton field rather than before.) If we now 'cut out' a volume $L^{4}$ centered on the instanton and impose periodicity on that volume, there will be only a small mismatch at the boundaries as long as $L \gg \rho$. To smoothen this approximate lattice instanton we typically perform 5 cooling sweeps upon it. As long as $L \gg \rho \gg 1$ the size of the instanton will not change significantly and this final field is, to a good approximation, a periodic lattice instanton of size $\rho$. (In practice one finds that the cooling does not appreciably alter the size of the inatanton as long as $\rho \geq 2$.)

The Maximally Abelian (MA) gauge is defined as the gauge in which the 'adjoint' operator

$$
X(n)=\sum_{\mu}\left\{U_{\mu}(n) \sigma_{3} U_{\mu}^{\dagger}(n)+U_{\mu}^{\dagger}(n-\widehat{\mu}) \sigma_{3} U_{\mu}(n-\widehat{\mu})\right\}
$$

points in the $\sigma_{3}$ direction for all $n$. Since this operator depends on gauge transformations in a non-local way this can only be achieved by an iterative site-by-site method which is made more efficient by incorporating over-relaxation [11]. Once $X(n)$ has been made proportional to $\sigma_{3}$ there is a remnant $U(1)$ symmetry which leaves this invariant and this defines for us our $U(1)$ fields. These fields will generically contain topological singularities that are magnetic monopoles. The 
corresponding magnetic 4-currents on the dual Abelian lattice can be identified [12] and resolved into separate closed monopole world-lines. (This last step is largely unambiguous in our case, since typically only $1-2 \%$ of the dual links carry a non-zero monopole current.)

We have constructed lattice instantons of various sizes and on various lattice volumes using the above construction. When we put these fields into the MA gauge, we find that each of the corresponding U(1) fields contains a single monopole loop (as long as the instantons are not too small: $\rho \geq 2$ ). This loop lies within the core of the instanton and its length is an increasing function of the instanton core size, although to see this one must be on lattices that are sufficiently large; see Table 1. Moreover the loop tends to be planar on the smaller lattices and for the smaller values of $\rho$. As one would expect, the centroid of the loop (nearly) coincides with the centre of the instanton as measured by the maximum of $Q(n)$.

Of course the above results have been obtained for the initial instanton configuration in a very special gauge. As we have previously remarked, the extracted $U(1)$ fields differ between differing Gribov copies and so it is possible that what we have seen so far is not characteristic of general instanton fields. To investigate this question we generate more general instanton fields by applying random $S U(2)$ local gauge transformations to the lattice instantons of eqns(1-4). When we do so we once again find the characteristic feature that each such field possesses a single monopole loop (if the instanton is not too small). There are, however, variations in the lengths and shapes of the loops and these variations become larger for larger lattices and larger instantons. In Table 2 we present the average loop lengths and the standard deviations as obtained from 5 such randomly gauged instanton fields for each instanton and lattice size. We can infer that for a fixed instanton size the loop length becomes independent of the lattice size, $L$, once $L \geq 2 \rho$. We also see that this loop length grows with $\rho$ and that this growth is roughly linear. This is not unexpected given the scale-invariance of the continuum gauge theory in an infinite volume.

At this stage we have established what occurs when we have a single classical instanton. If this is to be of relevance to the quantum field theory, we must show that what we have seen so far is in fact robust under the inclusion of, at the very least, small quantum fluctuations. To address this question we need to incorporate such fluctuations around the instanton field. To do so we start with our usual classical lattice instanton field and then perform a few Monte Carlo sweeps at some value of $\beta$. The number of sweeps needs to be chosen large enough that perturbative fluctuations are accurately incorporated (in the sense that the plaquette is close to its equilibrium value) while being small enough that the background instanton field is essentially unchanged (as can be checked by cooling the final configuration). The value of $\beta$ is chosen so that the lattice volume being used is sufficiently small that we do not run the risk of generating other large-scale non-perturbative fluctuations. The results we present here have actually been obtained using 5 Monte Carlo sweeps at $\beta=3.0$ on a $16^{4}$ lattice.

We once again find that an instanton is always associated with a monopole loop located within the core of the instanton. We show in Fig. 1 how the average loop length varies with instanton size. The errors are based on 5 different field configurations at each instanton size and so should only be taken as indicative of the true errors. Nonetheless we find clear evidence for a linear 
dependence between instanton size and loop length, even in the presence of quantum fluctuations which ultimately break the scale-invariance of the theory. The loop length is typically much longer than for the corresponding classical case, as we see if we compare Table 2 and Fig 1, and this seems to be primarily because the perimeter of the loop is much less smooth. In addition to the loop associated with the monopole, there are typically several much smaller loops in the vacuum - and this is so even if we have no instanton. Such small loops will, however, play no role in affecting phenomena on physical length scales. If the field is now cooled then the loop shrinks back close to its characteristic classical length.

By now it should be clear that our results differ from those of [5]. This is no surprise since that solution had to be special to a strictly infinite volume. The reason is that any 3-volume orthogonal to the monopole world line found in [5] contains just a single static monopole and, by the conservation of magnetic flux, this would not be possible on a finite periodic volume. A contractible monopole loop, in contrast, will correspond to a monopole-antimonopole pair in any intersecting 3-volume.

\section{Interacting (Anti-)Instantons}

The real vacuum will contain a number of topological charges that is proportional to the volume. If the separations between these charges were large compared to the typical core size, then the calculations in the previous section would imply that the corresponding $U(1)$ fields would consist of a dilute gas of monopole loops centered on these charges, with sizes comparable to the sizes of the corresponding cores. However in the real world the typical core size and typical inter-core separation must be comparable because the theory contains only one scale. In this case what happens to the monopole loops? In particular do they form much larger loops and so affect the confining properties of the theory?

To approach this question we first consider the simpler case of an instanton and an (anti)instanton, each of size $\rho$ and separated by a distance $\delta$. Such a configuration can be created by joining two hypercubic sub-lattices on which instanton potentials have been separately constructed, and performing say five cooling sweeps to smoothen any mismatch at the boundaries. This method fails if $\delta$ is too small as compared to $\rho$ - one finds that the end result is only a single instanton (as one might expect). Similarly for an instanton and anti-instanton.

We begin with topological charges constructed to be oriented along the 4-direction in group space, as in eqns $(1,2)$, and on sub-lattices arranged along the 4-direction of space. After projecting to the MA gauge, we find that if $\delta$ is small we do not have 2 separate monopole loops but rather a single larger loop that encloses both centres. The length is too great to be accounted for as a simple superposition of two separate loops. The separation below which this occurs is substantially less for pairs of like charge than for ones of opposite charge [see Table 3]. For like pairs the critical separation at which the two individual monopole loops merge is about equal to the core size while for unlike pairs it is about twice as large. Different Gribov copies (obtained by performing random gauge transformations on the fields just before MA gauge fixing) do not differ in any essentials: a 
large loop remains large although its plane may rotate around the axis joining the centres of the instanton and (anti-)instanton.

In the real vacuum the relative orientations in real and group spaces of neighbouring topological charges will not be the same, although they may well be correlated, partly through their interactions (the action will depend on the relative orientation) and partly through the fact that they will be embedded within other nonperturbative fluctuations. We have therefore investigated what happens under changes in both the orientation in group space and relative positioning of, say, an antiinstanton relative to that of the neighbouring instanton. We have studied the five different relative positionings of the sub-lattices along the principal lattice axes, with rotations of the anti-instanton solution relative to that of the instanton also about these axes. We focus on the interesting case where $\delta$ is small enough to produce a single large monopole loop for at least some orientations. For the case described in the previous paragraph,we find that for rotations up to about $\pi / 6$ the loop does not change. For larger angles it begins to twist near the centre. It is only once the angle becomes greater than about $2 \pi / 3$, however, that the monopole loop breaks into two separate loops, each centered on one of the two topological charges [see Figure 2]. Similar angle dependent loop structure is seen in two more of the five cases, which makes it reasonable to infer that for completely general positioning and rotation, which we have not investigated, nearby topological charges have a substantial probability to form a single, large loop rather than two smaller ones.

We have carried out a less complete investigation for cases of more instantons. By creating a linear stack of three sub-lattices containing alternate instanton and anti-instanton solutions with equal separations of centres, we find that for rotations of each sublattice by different angles (although about parallel axes) there are orientations for which a large single loop enclosing all three centres is formed rather than three single loops. For all five relative positions and rotations the formation of this appears to be governed by the relative orientations of nearest neighbour charges only, and depends upon this in accordance with the pair-wise interactions discussed above. Similar results are seen for linear stacks of four alternating instanton charges. By placing 4 sub-lattices together we obtain a $2 \times 2$ chequer-board of instantons and anti-instantons and we then find cases of two mutual loops each enclosing a separate pair of neighbouring, opposite charges, or of one large loop enclosing all four. The latter case occurs when all of the four possible instanton-anti-instanton pairs have relative orientations consistent with formation of a mutual loop in the pair-wise interaction. The former when this is so for only two such pairs. A few trial cases of $2 \times 2 \times 2$ and $2 \times 2 \times 2 \times 2$ chequer-boards of opposite charges yielded loops that could similarly be explained in terms of pair-wise interactions of neighbouring charges although in these cases we have not yet seen cases where there is only a single large monopole loop.

The above calculations suggest that if the instanton gas is moderately dense, as one would expect for a theory with one scale, then the monopole loops that would have been associated with the individual topological charges might merge into a smaller number of larger loops. This would seem to require a rather specific ordering of neighbouring instanton orientations. Such large loops, if formed, would be interesting because they could have an impact on the confining properties of the theory. Clearly a systematic study of the multi-instanton case, including quantum fluctuations, would be worth carrying out and we intend to do so. 


\section{The $S U(2)$ Vacuum}

So far we have investigated the connection between monopoles and instantons in fields where the former are the only non-perturbative fluctuations. Eventually, however, we would like to understand what happens in the fully quantised vacuum. We therefore include here a brief and far from systematic study of the real gauge vacuum.

To do so we take a typical $S U(2)$ field configuration on a $16^{4}$ lattice generated at $\beta=2.5$. (Such a lattice is moderately large in physical units.) We put this field into the MA gauge and extract the corresponding monopole loops. We then perform a sequence of cooling sweeps on the $S U(2)$ field. This rapidly removes the high frequency fluctuations and exposes the topological structure. (If the lattice were arbitrarily large and the lattice spacing arbitrarily small then the cooling would eventually produce a multi-instanton configuration corresponding to the minimum of the action.) As we cool, we put the cooled configuration into the MA gauge and extract the corresponding monopole loops. In Table 1 we show how the number of loops and their average length varies with the number of $S U(2)$ cooling sweeps. After the second cooling sweep there are only 3 loops. Two are relatively small and they lie within the two pronounced (because rather narrow) topological charges in the cooled field. The third loop is very long (initially about 220 links), extends over the whole lattice and shrinks only gradually with further cooling. There are a number of broader, overlapping instantons and anti-instantons on the lattice at this stage of cooling and since there are no individual monopole loops associated with them, it must be that these loops have joined into a much larger loop (as in the previous section) and that this is just our third loop. If we now continue cooling then we find that somewhere between 10 and 15 cooling sweeps one of the narrowish instantons shrinks out of the lattice and the corresponding small monopole loop disappears. Eventually the other narrow instanton shrinks out of the lattice and only one loop remains. By the time we have performed 150 cooling sweeps our field contains only one instanton and what was our very long third loop is the only loop present, being now 34 links long and within the core of the remaining instanton.

This kind of study (we have carried out additional examples) demonstrates in a direct way that while the smallest instantons will be associated with small monopole loops, the monopole loops associated with the overlapping topological charges of a more typical size do in fact combine into very large loops that extend over much of the lattice. Of course it may be that they have combined to form only part of the large loop in our example: it might be that there are other non-perturbative fluctuations that also contribute to the formation of this loop. One might object that after a few cooling sweeps this would be an unnecessary qualification. However this is not so. To test this hypothesis we have cooled $S U(2)$ fields in $2+1$ dimensions where there are no non-trivial minima of the action and where under the cooling the lattice action smoothly descends to zero. In that case we find it common for cooled fields to possess distant monopole-antimonopole pairs even after 10 cooling sweeps. This tells us that we cannot assume that on the cooled lattices any monopole loops must be of topological origin.

Our ultimate interest is, of course, in the monopole loops associated with the original uncooled lattice field. We have focussed on the cooled lattices because from these we can learn what kind of 
monopole loops are associated with the topological structure of the fields. Having done so, we can ask about the nature of those loops that are not topological in origin. Our particular interest is with the very extended monopole loops that will disorder large Wilson loops and hence contribute to confinement. Now, as we see from the first column in Table 4 , the original configuration contained a large number of loops. Most of these immediately disappeared under cooling. In addition this included some very long loops. Of course it is possible that some loops are long not because they are very extended but because they are very crumpled and in that case they would not contribute to confinement. To address this question in a simple manner we introduce a method to smoothen the $U(1)$ fields. The simplest technique is to perform $U(1)$ cooling in a manner entirely analogous to the $S U(2)$ cooling. We sweep through the $U(1)$ lattice field minimising, say, the plaquette action. This rapidly removes the high frequency fluctuations of the $U(1)$ fields. Any kinks and crumpling in the monopole loops is removed leaving large smooth monopole loops that are nearly stable under further $U(1)$ cooling. In Table 4 we show the number of monopole loops and their average length as a function of the number of $U(1)$ cooling sweeps performed on the $U(1)$ field obtained from the original fully quantised $S U(2)$ field. As we see from the Table most of the original loops must be either small or crumpled because they disappear almost immediately under the $U(1)$ cooling. There are, however, several very large loops remaining, in contrast to the single large loop associated with the topological structure, and so it is clear that most of the very large monopole loops that can be important for confinement, do not in fact have a topological origin. This conclusion has been reinforced by the study of further cases than the one described in detail here.

\section{Conclusions}

We have shown that for cases where the only non-perturbative fluctuation in the $S U(2)$ fields is an isolated instanton, the $U(1)$ field obtained by going to the Maximally Abelian gauge contains a single monopole loop within the core of the instanton. The size of the loop is proportional to the core size. We have also studied instanton (anti)instanton pairs as a function of their separation. For large separations these simply produce two individual loops and from this we can infer that a dilute gas of (anti)instantons will produce a correspondingly dilute gas of finite monopole loops. Such a gas of loops would not affect the confining properties of the theory, since that depends on the presence of a suitable distribution of arbitrarily large loops. On the other hand we find that for reasonably small separations, the loops typically do combine into larger loops and this raises the possibility that a moderately dense gas of topological charges, of the kind that one would expect to be present in the real vacuum, could produce very extended loops. Our study of examples of typical vacuum fields suggested that while this indeed seemed to be the case, only a fraction of the very large loops that drive Abelian confinement, have a topological origin. It is also the case that our calculations suggest that the formation of such large loops from the background topological structure requires that the correlation length characterising the relative orientations (in group space) of nearby instantons should not be too small. If these correlations are produced by vacuum fluctuations other than instantons, as they probably have to be, then the association of the resulting very large loops with the topological structure becomes less causal. 
We finish with some speculative remarks on the implications of this for the physics of the vacuum. Firstly, if, as we have seen, the topological structure of the $S U(2)$ vacuum typically produces monopole loops that extend over the whole space-time volume, then it contributes to the linear confining potential. This contradicts what is found with direct calculations in instanton gas and liquid models. The solution to this puzzle probably lies in our observation that the formation of large loops requires strong correlations between the orientations, in group space, of neighbouring charges. This would not occur in an instanton gas or liquid and probably requires the presence of other non-perturbative fluctuations.

Our second speculation concerns chiral symmetry breaking. A fermion in the field of a static monopole has a zero energy mode [13]. Thus one expects that a large monopole loop should be associated with a small eigenvalue of the Dirac operator, with this eigenvalue going to zero as the size of the loop grows to infinity. So large loops that extend throughout the lattice could be associated with a distribution of modes that extends to zero. On the other hand in current lattice calculations one finds that it is the topological fluctuations of the $S U(2)$ fields that produce the small modes that spontaneously break chiral symmetry [7]. So it would be natural if in the $U(1)$ fields it was the large monopole loops associated with topology that provided the symmetry breaking spectrum of near-zero modes. This is an idea that could be tested explicitly. It certainly fits in with recent work 14] showing that the $U(1)$ fields do possess a symmetry breaking eigenvalue spectrum of the Dirac operator.

\section{Acknowledgements}

An earlier version of this work was presented at the Workshop on Non-Perturbative QCD at ECT (Trento) this summer and we are grateful for very useful discussions with participants there. One of us (AGH) acknowledges the support of PPARC studentship 93300888. The calculations were supported by PPARC Grants GR/J21408 and GR/K55752.

\section{References}

[1] G. 't Hooft — Nucl. Phys. B190 (1981) 455.

[2] T. Suzuki and I. Yotsuyanagi — Phys. Rev. D 42 (1990) 4257.

[3] A.S. Kronfeld, M.L. Laursen, G. Schierholz and U.-J. Wiese — Phys. Lett. 198B (1987) 516.

[4] T. Suzuki - Nucl. Phys. B30 (Proc.Suppl.) (1993) 176.

[5] M.N. Chernodub and F.V. Gubarev — Preprint ITEP-95-34 available as hep-th/9506026.

[6] D. Diakonov - Lectures at the Enrico Fermi School in Physics, Varenna (1995).

[7] S.J. Hands and M. Teper — Nucl. Phys. B347 (1990) 819.

[8] J. Hoek, M. Teper and J. Waterhouse — Nucl. Phys. B288 (1987) 589. 
[9] A.A. Belavin, A.M. Polyakov, A.S. Schwartz and Yu.S. Tyupkin - Phys. Lett. 59B (1975) 85.

[10] M. Teper - Oxford Preprint OUTP-59/88 (unpublished).

[11] J.E. Mandula and M. Ogilvie — Phys. Lett. 248B (1990) 156.

[12] T.A. DeGrand and D. Toussaint — Phys. Rev. D 22 (1980) 2478.

[13] P. Osland and T.T. Wu - Nucl. Phys. B247 (1984) 421.

[14] F.X. Lee, R.M. Woloshyn and H.D. Trottier - Preprint TRI-PP-95-62 available as heplat/9509028. 


\begin{tabular}{|c||c|c|c|c|}
\hline \multicolumn{1}{|c||}{ one $i$} & \multicolumn{4}{c|}{ Lattice size } \\
\cline { 2 - 5 }$\rho$ & $8^{4}$ & $12^{4}$ & $16^{4}$ & $24^{4}$ \\
\hline \hline 2.0 & 8 & 4 & 0 & - \\
2.5 & 8 & 8 & 8 & - \\
3.0 & 12 & 8 & 8 & - \\
3.5 & 16 & 8 & - & - \\
4.0 & 8 & 8 & 8 & 16 \\
5.0 & 16 & 16 & 8 & - \\
6.0 & 10 & 16 & 16 & 16 \\
7.0 & 8 & 16 & 16 & - \\
8.0 & 8 & 16 & 16 & 24 \\
9.0 & 8 & 16 & - & - \\
10.0 & 8 & 16 & 16 & 24 \\
12.0 & - & - & - & 24 \\
14.0 & - & - & - & 32 \\
\hline
\end{tabular}

Table 1: Lengths of monopole loops for various instanton and lattice sizes, using the particular solution of equations $(1,2)$ ( - denotes this case unstudied).

\begin{tabular}{|c||c|c|c|c|c|}
\hline \multicolumn{1}{|c||}{ one $i$} & \multicolumn{5}{c|}{ Lattice size } \\
\cline { 2 - 6 }$\rho$ & $6^{4}$ & $8^{4}$ & \multicolumn{1}{c|}{$12^{4}$} & $16^{4}$ & \multicolumn{1}{c|}{$24^{4}$} \\
\hline \hline 2.0 & $8.0(0)$ & $8.0(0)$ & $2.4(2.2)$ & $0(0)$ & - \\
2.5 & $8.4(0.9)$ & $8.0(0)$ & $8.4(0.9)$ & $8.0(0)$ & - \\
3.0 & $9.6(0.9)$ & $11.6(0.9)$ & $8.8(1.1)$ & $8.8(1.8)$ & - \\
4.0 & $8.0(0)$ & $14.4(2.2)$ & $12.0(2.5)$ & $10.8(1.1)$ & $10.4(2.2)$ \\
5.0 & $8.0(0)$ & $13.2(2.3)$ & $15.2(1.1)$ & $16.0(0)$ & - \\
6.0 & $8.0(0)$ & $11.2(2.1)$ & $16.0(1.1)$ & $15.2(1.8)$ & $18.0(7.9)$ \\
7.0 & - & $9.6(1.7)$ & $15.2(1.8)$ & $18.4(0.9)$ & - \\
8.0 & - & $8.0(0)$ & $18.4(0.9)$ & $19.6(0.9)$ & $18.4(3.6)$ \\
9.0 & - & - & $19.6(0.9)$ & $21.2(2.7)$ & - \\
10.0 & - & - & $21.2(0.7)$ & $22.4(0.9)$ & $34.8(17.2)$ \\
11.0 & - & - & $22.4(0.9)$ & $22.4(2.6)$ & - \\
12.0 & - & - & $22.4(2.6)$ & - & $51.2(24.8)$ \\
14.0 & - & - & - & - & $51.6(11.9)$ \\
\hline
\end{tabular}

Table 2: Lengths of monopole loops associated with instantons of size $\rho$ in various lattice volumes: means (standard deviations). 


\begin{tabular}{|c||c|c|}
\hline \multicolumn{1}{|c||}{$i-i$} & \multicolumn{2}{c|}{ Lattice size } \\
\cline { 2 - 3 }$\delta$ & $8^{3} .16$ & $12^{3} .24$ \\
\hline \hline 12.0 & - & 8,8 \\
8.0 & - & 8,8 \\
7.0 & - & - \\
6.0 & 8,8 & - \\
5.0 & 8,8 & 8,8 \\
4.0 & 8,8 & 8,8 \\
3.0 & 12 & 12 \\
2.0 & - & 12 \\
\hline
\end{tabular}

\begin{tabular}{|c||c|c|}
\hline \multicolumn{1}{|c||}{$i-\bar{\imath}$} & \multicolumn{2}{c|}{ Lattice size } \\
\cline { 2 - 3 }$\delta$ & $8^{3} .16$ & $12^{3} .24$ \\
\hline \hline 12.0 & - & 8,8 \\
8.0 & 8,8 & - \\
7.0 & 8,8 & 8,8 \\
6.0 & 8,8 & 24 \\
5.0 & 18 & 18 \\
4.0 & 16 & 20 \\
3.0 & 10 & 10 \\
2.0 & 0 & 0 \\
\hline
\end{tabular}

Table 3: Lengths of monopole loops for (a) like and (b) unlike pairs of charges with $\rho=3$.

\begin{tabular}{|c||c|c|c|c|c|c|c|c|c|c|}
\hline$S U(2)$ Cools & 0 & 1 & 2 & 3 & 4 & 5 & 10 & 15 & 20 & 150 \\
\hline \hline No. loops & 366 & 10 & 3 & 3 & 3 & 3 & 3 & 2 & 2 & 1 \\
\hline Mean length & 9.7 & 42.0 & 82.7 & 78.0 & 75.3 & 74.7 & 64.0 & 85.0 & 81.0 & 34 \\
\hline
\end{tabular}

\begin{tabular}{|c||c|c|c|c|c|c|c|c|c|c|}
\hline$U(1)$ Cools & 0 & 1 & 2 & 3 & 4 & 5 & 10 & 15 & 20 & 150 \\
\hline \hline No. loops & 366 & 23 & 17 & 16 & 14 & 11 & 9 & 8 & 8 & - \\
\hline Mean length & 9.7 & 50.7 & 56.7 & 54.6 & 56.7 & 67.1 & 67.6 & 68.3 & 62.3 & - \\
\hline
\end{tabular}

Table 4: Number of loops and average loop length of one typical configuration under cooling. 


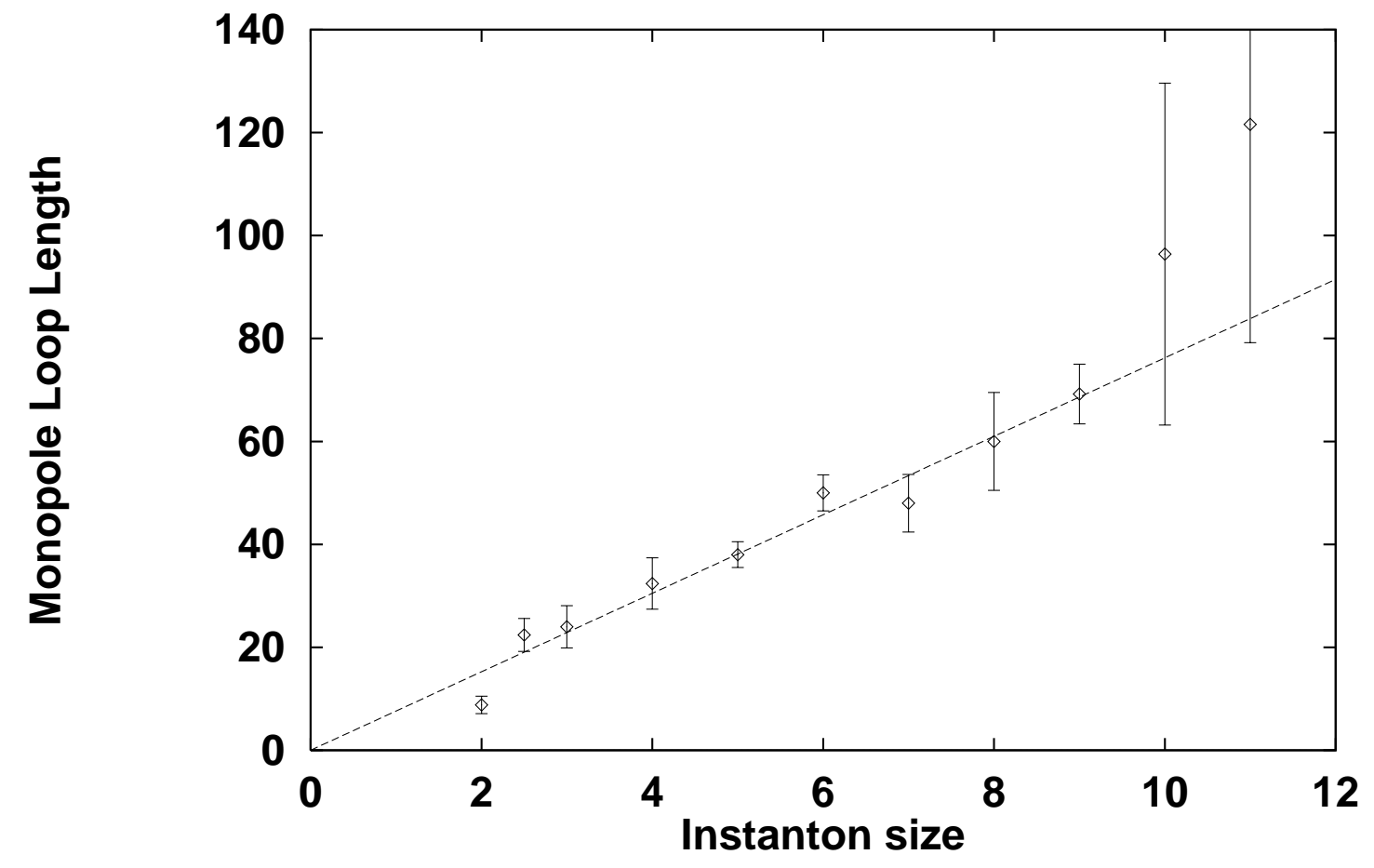

Figure 1: Monopole loop length versus instanton size for instantons, including quantum fluctuations, on a $16^{4}$ lattice. 
0 deg.

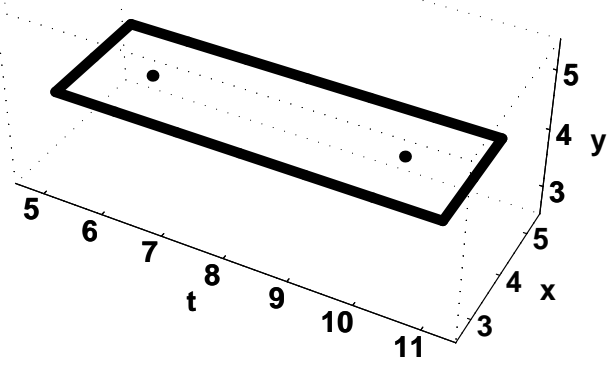

$112.5 \mathrm{deg}$.

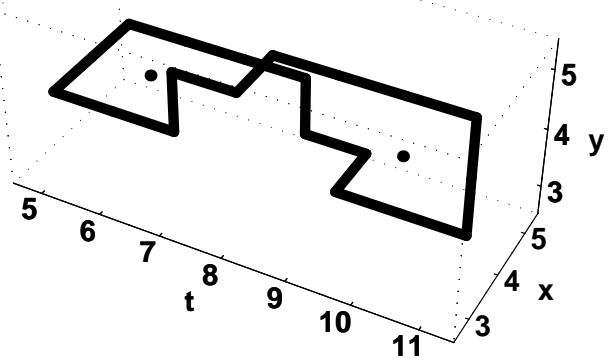

90 deg.

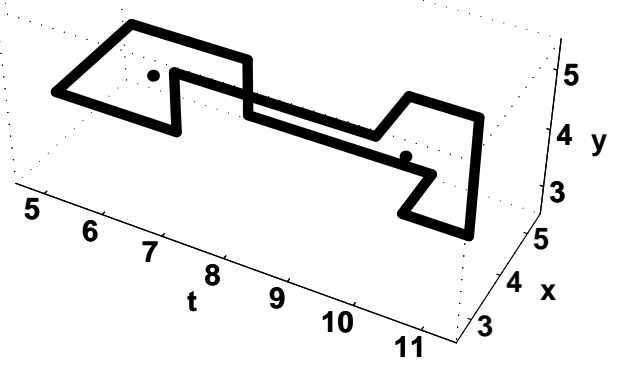

$120 \mathrm{deg}$.

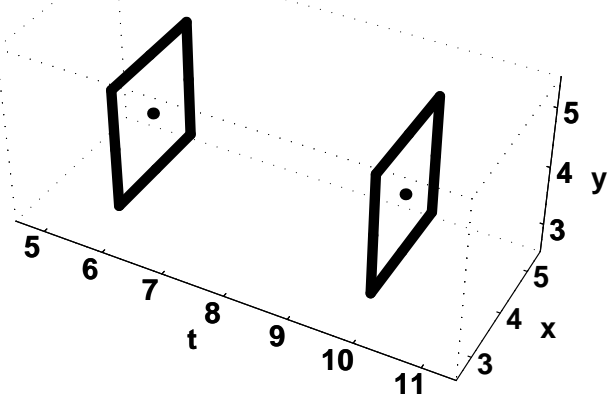

Figure 2: Three dimensional projections of the mutual monopole loop surrounding an instanton-anti-instanton pair (centres marked) of size $\rho=3$ under increasing rotation angle as detailed in the text. The loops are flat in the fourth direction. 